\title{
Loss of Transient Receptor Potential Melastatin 3 ion channel function in natural killer cells from Chronic Fatigue Syndrome/ Myalgic Encephalomyelitis patients
}

Hélène Cabanas ${ }^{1,2^{*}}$ (D) Katsuhiko Muraki ${ }^{3}$, Natalie Eaton ${ }^{1,2}$, Cassandra Balinas ${ }^{1,2}$, Donald Staines ${ }^{1,2}$ and Sonya Marshall-Gradisnik ${ }^{1,2}$

\begin{abstract}
Background: Chronic Fatigue Syndrome (CFS)/ Myalgic Encephalomyelitis (ME) is a debilitating disorder that is accompanied by reduced cytotoxic activity in natural killer (NK) cells. NK cells are an essential innate immune cell, responsible for recognising and inducing apoptosis of tumour and virus infected cells. Calcium is an essential component in mediating this cellular function. Transient Receptor Potential Melastatin 3 (TRPM3) cation channels have an important regulatory role in mediating calcium influx to help maintain cellular homeostasis. Several single nucleotide polymorphisms have been reported in TRPM3 genes from isolated peripheral blood mononuclear cells, NK and B cells in patients with CFS/ME and have been proposed to correlate with illness presentation. Moreover, a significant reduction in both TRPM3 surface expression and intracellular calcium mobilisation in NK cells has been found in CFS/ME patients compared with healthy controls. Despite the functional importance of TRPM3, little is known about the ion channel function in NK cells and the epiphenomenon of CFS/ME. The objective of the present study was to characterise the TRPM3 ion channel function in NK cells from CFS/ME patients in comparison with healthy controls using whole cell patch-clamp techniques.
\end{abstract}

Methods: NK cells were isolated from 12 age- and sex-matched healthy controls and CFS patients. Whole cell electrophysiology recording has been used to assess TRPM3 ion channel activity after modulation with pregnenolone sulfate and ononetin.

Results: We report a significant reduction in amplitude of TRPM3 current after pregnenolone sulfate stimulation in isolated NK cells from CFS/ME patients compared with healthy controls. In addition, we found pregnenolone sulfate-evoked ionic currents through TRPM3 channels were significantly modulated by ononetin in isolated NK cells from healthy controls compared with CFS/ME patients.

Conclusions: TRPM3 activity is impaired in CFS/ME patients suggesting changes in intracellular $\mathrm{Ca}^{2+}$ concentration, which may impact NK cellular functions. This investigation further helps to understand the intracellular-mediated roles in NK cells and confirm the potential role of TRPM3 ion channels in the aetiology and pathomechanism of CFS/ME.

Keywords: Transient receptor potential Melastatin 3, Calcium, Chronic fatigue syndrome/Myalgic encephalomyelitis, Natural killer cells, Flow cytometry, Patch-clamp

\footnotetext{
* Correspondence: h.cabanas@griffith.edu.au

${ }^{1}$ School of Medical Science, Griffith University, Gold Coast, QLD, Australia

${ }^{2}$ The National Centre for Neuroimmunology and Emerging Diseases, Menzies

Health Institute Queensland, Griffith University, Gold Coast, QLD, Australia

Full list of author information is available at the end of the article
}

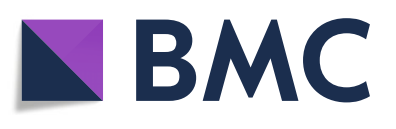

(c) The Author(s). 2018 Open Access This article is distributed under the terms of the Creative Commons Attribution 4.0 International License (http://creativecommons.org/licenses/by/4.0/), which permits unrestricted use, distribution, and reproduction in any medium, provided you give appropriate credit to the original author(s) and the source, provide a link to the Creative Commons license, and indicate if changes were made. The Creative Commons Public Domain Dedication waiver (http://creativecommons.org/publicdomain/zero/1.0/) applies to the data made available in this article, unless otherwise stated. 


\section{Background}

Chronic Fatigue Syndrome/ Myalgic Encephalomyelitis (CFS/ME) is a complex and debilitating disorder hallmarked by persistent or relapsing chronic fatigue that is inadequately alleviated by rest (Fukuda et al., 1994). At least four concurrent symptoms related to multiple systems including the immune, neurological, musculoskeletal, gastrointestinal, and cardiovascular systems accompany this unexplained fatigue (Carruthers et al., 2011). Without a pathology test, diagnosis is complex and relies on different case definitions that address the characteristics of CFS/ME (Carruthers et al., 2011; Jason et al., 2015). Although the Centers for Disease Control and Prevention (CDC) criteria are widely employed, this definition has been considered too broad in its symptom requirements (Johnston et al., 2013). Thus, the definition of CFS/ME was revised producing the Canadian Consensus Criteria (CCC) and finally in 2011 a more specific definition was established known as the International Consensus Criteria (ICC) to assist diagnosis (Carruthers et al., 2011). The underlying aetiology of CFS/ME remains unknown. However, a consistent feature of CFS/ $\mathrm{ME}$ in the literature is immune dysfunction, and more precisely a significant reduction in Natural Killer (NK) cell cytotoxicity, a hallmark of NK cell function (Brenu et al., 2012; Brenu et al., 2011; Curriu et al., 2013; Hardcastle et al., 2015; Huth et al., 2016a; Klimas et al., 1990; Maher et al., 2005; Natelson et al., 2002; Nijs \& Frémont, 2008; Sharpe et al., 1991; Siegel et al., 2006; Stanietsky \& Mandelboim, 2010).

NK cells are effector lymphocytes of the innate immune system that eliminate pathogens and tumour cells, in addition to immune cell activation and cytokine production (Vivier et al., 2008). NK cells can be divided into five different phenotypes determined by their expression of cell-surface molecules including CD56 and CD16. The two main mature NK cell subtypes are $\mathrm{CD} 56^{\text {bright }} \mathrm{CD} 16^{\mathrm{dim}}$ and CD56 ${ }^{\text {dim }} \mathrm{CD} 16^{\text {bright }}$. CD56 ${ }^{\text {bright }} \mathrm{CD} 166^{\mathrm{dim}}$ NK cells exist as the minority in peripheral blood as efficient cytokine producers (Cooper et al., 2001). The CD56 ${ }^{\mathrm{dim}} \mathrm{CD} 16^{\text {bright }}$ subset constitutes $90 \%$ of human peripheral NK cells with significantly higher cytotoxic activity than CD56 $6^{\text {bright }}$ NK cells as they contain an abundance of cytolytic proteins. Additionally, the presence of the low-affinity $\mathrm{Fc}_{\mathrm{C}-} \gamma$ receptor $\mathrm{CD} 16 \mathrm{fa}$ cilitates the activation of antibody-dependent cellular cytotoxicity (ADCC) (Moretta, 2010; Stabile et al., 2015). Differences in NK cell phenotypes and significantly reduced peripheral NK cell numbers resulting in significant reduction in NK cell cytotoxicity, have been reported in CFS/ME and implicated in disease severity (Brenu et al., 2013; Hardcastle et al., 2015; Lanier, 2003; Maher et al., 2005; Nijs \& Frémont, 2008). Importantly, NK cells require calcium $\left(\mathrm{Ca}^{2+}\right)$ to regulate cellular functions including NK cell cytotoxicity. Indeed, numerous steps including adhesion to the target cell, activation of surface receptors, microtubule reorganisation, polarisation of secretory granules and release of lytic proteins, including granzyme A and granzyme B, creation of the immune synapse, formation of perforin pores, and finally granzyme-induced target cell apoptosis require tight regulation of $\mathrm{Ca}^{2+}$ signalling (Anasetti et al., 1987; Henkart, 1985; Kass \& Orrenius, 1999; Schwarz et al., 2013).

Transient Receptor Potential (TRP) ion channels can trigger specific $\mathrm{Ca}^{2+}$-dependent signal transduction pathways regulating many biological processes in both excitable and nonexcitable cells (Gees et al., 2010). TRP channels represent a large and diverse family of nonselective cation channels that are widely expressed and respond to a wide range of chemical and physical stimuli (Voets et al., 2005). Moreover, genetic variations in TRP genes and noxious stimuli have been implicated in several pain-related pathological conditions/modalities, including inflammatory, neuropathic, visceral and dental pain, as well as pain associated with cancer (Mickle et al., 2016; Nilius, 2007). The mammalian TRP channels are divided into subgroups according to amino acid sequence similarities: TRPC (canonical), TRPM (melastatin), TRPV (vanilloid), TRPA (ankyrin), TRPML (mucolipin), and TRPP (polycystin) (Clapham et al., 2001). TRP cation channel subfamily $M$ member 3 (TRPM3) is a $\mathrm{Ca}^{2+}$-permeable nonselective cation channel, expressing a calmodulin binding region on the $\mathrm{N}$-terminus which plays a role in activation of $\mathrm{Ca}^{2+}$ dependent signalling pathways (Holakovska et al., 2012; Lee et al., 2003; Oberwinkler \& Philipp, 2014). The human TRPM3 gene encodes for many different TRPM3 isoforms due to alternative splicing and exon usage, leading to channels with divergent pore and gating properties (Frühwald et al., 2012; Oberwinkler et al., 2005; Thiel et al., 2013). In particular, the TRPM $3 \alpha 2$ isoform has been characterized as being highly permeable for $\mathrm{Ca}^{2+}$ and other divalent cations (Frühwald et al., 2012). TRPM3 ion channels are highly expressed in neurons of dorsal root ganglia, where they serve as thermosensitive channels implicated in the detection of noxious heat (Vriens et al., 2011). Furthermore, TRPM3 has been identified in a number of tissues and cell types, including pancreatic beta cells, brain, pituitary gland, eye, kidney, and adipose tissue, that serve many different functions (Hoffmann et al., 2010; Oberwinkler \& Philipp, 2014; Wagner et al., 2008). While expressed ubiquitously in mammalian cells, the roles and functions of TRPM3 have yet to be determined in immune cells and more particularly in NK cells, where TRPM3 has been previously identified without electrophysiological evaluation (Nguyen et al., 2017; Nguyen et al., 2016).

TRPM3 ion channels are quickly $(<100 \mathrm{~ms})$ and reversibly activated by a neuronal steroid, Pregnenolone 
sulfate (PregS) (Wagner et al., 2008). The precursor, pregnenolone is derived from cholesterol and sulphated in vivo for biological actions in the immune and central nervous systems. It is associated with memory and cognition, neuronal myelination, activation of neurotransmitter-gated channels, modulation of glutamate-nitric oxide-guanosine $3^{\prime}, 5^{\prime}$-(cyclic) phosphate pathways, maintenance of glucose and insulin homeostasis and the management of noxious stimuli (Harteneck, 2013; Nilius \& Voets, 2008). Stimulation of TRPM3 with PregS in pancreatic beta-cells induces an intracellular signalling cascade, involving a rise in intracellular $\mathrm{Ca}^{2+}$ concentration $\left(\left[\mathrm{Ca}^{2+}\right]_{\mathrm{i}}\right)$, activation of the protein kinases Raf and extracellular signal-regulated kinases (ERK), resulting in the regulation of different cellular processes and a change in gene expression pattern (Thiel et al., 2013). However, it is notable that concentrations of PregS were sometimes high $(30-100 \mu \mathrm{M})$, causing non-specific effects without TRPM3. On the other hand, a natural compound, deoxybezoin ononetin, has been identified as a selective and potent blocker of PregS-induced TRPM3 currents in TRPM3-expressing dorsal root ganglia neurones and TRPM3 transfected HEK293 cells (Straub et al., 2013). Therefore, both use of TRPM3 agonist and blocker are critical to identify the activity of TRPM3 currents in native cells.

Regulation and importance of TRPM3 channels in NK cells and the epiphenomenon of CFS/ME is relatively unknown. Five single nucleotide polymorphisms (SNPs) (rs6560200, rs1106948, rs12350232, rs11142822, rs1891301) have been identified in TRPM3 genes in CFS/ ME patients (Marshall-Gradisnik et al., 2016). A recent investigation characterising TRPM3 related responses in NK cells and B lymphocytes found a significant reduction in expression of TRPM3 on the NK cell surface in CFS/ME patients compared with healthy controls (HC) (Nguyen et al., 2016). Moreover, isolated NK cells from CFS/ME patients have impaired TRPM3 activity following PregS stimulation, resulting in impaired $\mathrm{Ca}^{2+}$ mobilisation and reduced NK cell cytotoxicity (Nguyen et al., 2017). These results strongly suggest the importance of TRPM3 in the pathophysiology of CFS/ME. However, as the electrophysiological characterisation of endogenous TRPM3 channels on isolated NK cells is lacking, we aimed to characterise TRPM3 channel currents using whole cell patch-clamp measurements in $\mathrm{HC}$ and CFS/ME patients after modulation with PregS and ononetin. This novel approach may help to understand the clinical importance of TRPM3 in the pathomechanism of CFS/ME.

\section{Methods}

\section{Participant recruitment}

Six CFS/ME patients and six age- and sex-matched HC were recruited using the National Centre for Neuroimmunology and Emerging Diseases (NCNED) research database.
Participants were screened using a comprehensive questionnaire corresponding with the $\mathrm{CDC}, \mathrm{CCC}$ and ICC case definitions. All six CFS/ME patients were defined by the CCC. HC reported no incidence of fatigue and were in good health without evidence of illness. Participants were excluded from this study if they reported history of smoking, autoimmune diseases, cardiac disease, diabetes or other co-morbidities.

Two of the six CFS/ME patients reported regular administration of non-steroidal anti-inflammatory for pain relief. No participants reported use of pharmacological agents that directly or indirectly influence TRPM3 or $\mathrm{Ca}^{2+}$ signalling.

This investigation was approved by the Griffith University Human Research Ethics Committee (HREC/15/QGC/63).

\section{Peripheral blood mononuclear cell isolation and natural killer cell isolation}

A total of $85 \mathrm{ml}$ of whole blood was collected in ethylendiaminetetraacetic acid (EDTA) tubes between 8:00 am and 12:00 am. Routine full blood analysis was performed within $4 \mathrm{~h}$ of collection for red blood cell count, white blood cell count and granulocyte cell count.

Peripheral blood mononuclear cells (PBMCs) were isolated from $80 \mathrm{ml}$ of whole blood by centrifugation over a density gradient medium (Ficoll-Paque Premium; GE Healthcare, Uppsala, Sweden) as previously described (Brenu et al., 2011; Munoz \& Leff, 2006). PBMCs were stained with trypan blue (Invitrogen, Carlsband, CA, USA) to determine cell count and cell viability. PBMCs were adjusted to a final concentration of $5 \times 10^{7}$ cells $/ \mathrm{ml}$ for NK cell isolation.

NK cells were isolated by immunomagnetic selection using an EasySep Negative Human NK Cell Isolation Kit (Stem Cell Technologies, Vancouver, BC, Canada). NK Cell purification was determined using flow cytometry. NK cells were incubated for $20 \mathrm{~min}$ at room temperature in the presence of CD56 FITC $(0.25 \mu \mathrm{g} /$ $5 \mu \mathrm{l})$ and CD3 PE Cy7 $(0.25 \mu \mathrm{g} / 20 \mu \mathrm{l})$ monoclonal antibodies (BD Bioscience, San Jose, CA, USA) as previously described (Nguyen et al., 2017).

\section{Whole cell electrophysiology recording}

Borosilicate glass capillaries with an outside diameter of $1.5 \mathrm{~mm}$ and inside diameter of $0.86 \mathrm{~mm}$ (Harvard Apparatus, Holliston, MA, USA) were used as patch pipettes. Pipette resistance when filled with pipette solution was 8$12 \mathrm{M} \Omega$. The pipettes were mounted on a CV203BU head-stage (Molecular Devices, Sunnyvale, CA, USA) connected to a 3-way coarse manipulator and a micro-manipulator (Narishige, Tokyo, Japan). Electrical signals were amplified and recorded using an Axopatch 200B amplifier and PClamp 10.7 software (Molecular Devices, Sunnyvale, CA, USA). Data were filtered at $5 \mathrm{kHz}$ and 
sampled digitally at $10 \mathrm{kHz}$ via a Digidata $1440 \mathrm{~A}$ analogue to digital converter (Molecular Devices, Sunnyvale, CA, USA). The voltage-ramp protocol was a step from a holding potential of $+10 \mathrm{mV}$ to $-90 \mathrm{mV}$, followed by a $0.1 \mathrm{~s}$ ramp to $+110 \mathrm{mV}$, before returning to $+10 \mathrm{mV}$ (repeated every $10 \mathrm{~s}$ ). The liquid junction potential between the pipette and bath solutions $(-10 \mathrm{mV})$ was corrected. A leak current component was not subtracted from the recorded currents. Electrode was filled with the intracellular pipette solution containing $30 \mathrm{mM} \mathrm{CsCl}, 2 \mathrm{mM} \mathrm{MgCl}_{2}$, $110 \mathrm{mM}$ L-Aspartic acid, $1 \mathrm{mM}$ EGTA, $10 \mathrm{mM}$ HEPES, $4 \mathrm{mM}$ ATP, $0.1 \mathrm{mM}$ GTP, adjusted $\mathrm{pH}$ to 7.2 with $\mathrm{CsOH}$ and osmolality of $290 \mathrm{mOsm} / \mathrm{L}$ with D-mannitol. The pipette solution was filtered using a $0.22 \mu \mathrm{m}$ membrane filter (Sigma-Aldrich, St. Louise, MO, USA), divided into aliquots and stored at $-20{ }^{\circ} \mathrm{C}$. Bath solution contained: $130 \mathrm{mM}$ $\mathrm{NaCl}, 10 \mathrm{mM} \mathrm{CsCl}, 1 \mathrm{mM} \mathrm{MgCl}, 1.5 \mathrm{mM} \mathrm{CaCl} 2 \mathrm{H}_{2} \mathrm{O}$, $10 \mathrm{mM}$ HEPES, adjusted $\mathrm{pH}$ to 7.4 with $\mathrm{NaOH}$ and osmolality $300 \mathrm{mOsm} / \mathrm{L}$ with $\mathrm{D}$-glucose. All reagents were purchased from Sigma-Aldrich, except for ATP and GTP that were purchased from Sapphire Bioscience. TRPM3 currents were stimulated by adding $100 \mu \mathrm{M}$ PregS (Tocris Bioscience, Bristol, UK) to the bath solution, whereas PregS-induced TRPM3 currents were blocked by adding $10 \mu \mathrm{M}$ ononetin (Tocris Bioscience, Bristol, UK). All measurements were performed at room temperature. The authors removed the possibility of chloride current involvement in TRPM3 assessment by using L-Aspartic acid in the intracellular pipette solution. Unstable currents were also removed from the analysis. This ensured only TRPM3 function was assessed.

\section{Statistical analysis}

Cytometry data was exported from FacsDiva v8.1 and analysed using SPSS v24 (IBM Corp, Version 24, Armonk, NY, USA) and GraphPad Prism v7 (GraphPad Software Inc., Version 7, La Jolla, CA, USA). Electrophysiological data were analysed using pCLAMP 10.7 software (Molecular Devices, Sunnyvale, CA, USA). Origin 2018 (OriginLab Corporation, Northampton, MA, USA) and GraphPad Prism v7 (GraphPad Software Inc., Version 7, La Jolla, CA, USA) were used for statistical analysis and data presentation. The Shapiro-Wilk test was used to determine population normality. Statistical comparison was performed using the Mann-Whitney U test (Table 1, Fig. 1 and Fig. 3e), and Fishers exact test (Fig. $3 g$ ), to determine any significant differences. Significance was set at $p<0.05$ and the data are presented as mean \pm SEM unless otherwise stated.

\section{Results}

Participant characteristics and blood parameters

A total of twelve age- and sex-matched participants were included for this investigation. Demographic and clinical
Table 1 Blood parameters and patient demographic

\begin{tabular}{|c|c|c|c|}
\hline & CFS/ME & $\mathrm{HC}$ & $P$ Value \\
\hline Age (years) & $42.5 \pm 6.05$ & $42.8 \pm 5.47$ & 0.872 \\
\hline \multicolumn{4}{|l|}{ Gender n(\%) } \\
\hline Male & $2(20 \%)$ & $2(20 \%)$ & 1.000 \\
\hline Female & $4(80 \%)$ & $4(80 \%)$ & \\
\hline BMI $\left(\mathrm{kg} / \mathrm{m}^{2}\right)$ & $23.54 \pm 0.54$ & $23.35 \pm 1.37$ & 0.240 \\
\hline \multicolumn{4}{|l|}{ SF-36 } \\
\hline Fatigue (\%) & $30.42 \pm 8.91$ & $77.92 \pm 6.25$ & 0.009 \\
\hline General Health (\%) & $31.25 \pm 5.97$ & $74.31 \pm 4.50$ & 0.004 \\
\hline Physical Functioning (\%) & $70.83 \pm 6.64$ & $93.33 \pm 4.77$ & 0.015 \\
\hline Role Physical (\%) & $20.83 \pm 9.64$ & $87.50 \pm 12.5$ & 0.009 \\
\hline Role Emotional (\%) & $59.38 \pm 9.24$ & $96.88 \pm 3.13$ & 0.004 \\
\hline Social Functioning (\%) & $37.5 \pm 5.59$ & $95.83 \pm 4.17$ & 0.002 \\
\hline Body Pain (\%) & $49.17 \pm 5.27$ & $84.17 \pm 8.41$ & 0.015 \\
\hline \multicolumn{4}{|l|}{ Pathology } \\
\hline White Cell Count $\left(\times 10^{9} / L\right)$ & $5.58 \pm 0.30$ & $7.13 \pm 0.49$ & 0.03 \\
\hline Lymphocytes (×10/L) & $1.99 \pm 0.2$ & $1.75 \pm 0.22$ & 0.423 \\
\hline Neutrophils $\left(\times 10^{9} / \mathrm{L}\right)$ & $3.03 \pm 0.23$ & $4.78 \pm 0.42$ & 0.01 \\
\hline Monocytes $\left(\times 10^{9} / \mathrm{L}\right)$ & $0.41 \pm 0.05$ & $0.39 \pm 0.06$ & 0.748 \\
\hline Eosinophils $\left(\times 10^{9} / \mathrm{L}\right)$ & $0.12 \pm 0.02$ & $0.18 \pm 0.06$ & 0.936 \\
\hline Basophils $\left(\times 10^{9} / \mathrm{L}\right)$ & $0.04 \pm 0.01$ & $0.04 \pm 0.002$ & 0.799 \\
\hline Platelet $\left(\times 10^{9} / \mathrm{L}\right)$ & $265.0 \pm 23.36$ & $266.17 \pm 15.70$ & 1.00 \\
\hline Red Cell Count $\left(\times 10^{12} / \mathrm{L}\right)$ & $4.63 \pm 0.09$ & $4.69 \pm 0.13$ & 0.63 \\
\hline Haematocrit & $0.41 \pm 0.01$ & $0.42 \pm 0.01$ & 0.624 \\
\hline Haemoglobin (g/L) & $135.0 \pm 3.52$ & $139.67 \pm 5.21$ & 0.687 \\
\hline
\end{tabular}

SF-36 scores were analysed using participant questionnaire responses. Results from routine full blood analysis in CFS/ME patients and HC. Data presented as mean \pm SD. Abbreviations: CFS/ME, chronic fatigue syndrome/myalgic encephalomyelitis; $H C$, healthy controls; BMI, body mass index

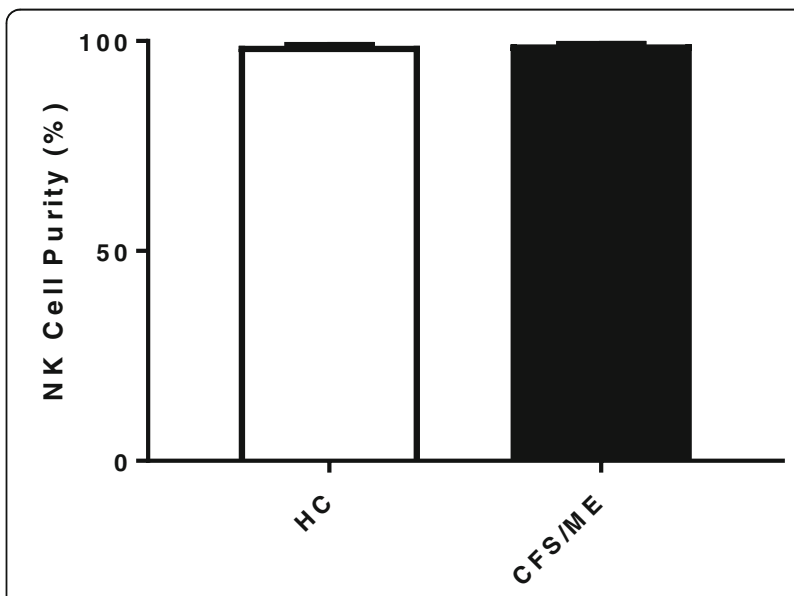

Fig. 1 Natural Killer cell purity. Bar graphs representing isolated NK cell purity for $\mathrm{HC}$ and CFS/ME patients. Data presented as mean \pm SEM. $\mathrm{HC}=98.07 \% \pm 0.80$ and $\mathrm{CFS} / \mathrm{ME}=98.28 \% \pm 0.58$. Abbreviations: CFS/ME, chronic fatigue syndrome/myalgic encephalomyelitis; $\mathrm{HC}$, healthy controls; NK cell, natural killer cell 
data for patients are summarised in Table 1. There was no significant difference in age or gender between patients and HC. The 36-Item Short Form Survey (SF-36) was used to determine participant health-related-quality of life. As expected, there was a significant difference in SF-36 scores between CFS/ME patients and HC. Moreover, while there was a significant difference in white cell count and neutrophils, all results remained within normal range for age and sex as provided by the Gold Coast University Hospital Pathology Unit, NATA accredited laboratory.

\section{Natural killer cell purity}

NK cell purity $\left(\mathrm{CD}^{-} / \mathrm{CD}^{+} 6^{+}\right)$was $98.07 \% \pm 0.80$ for $\mathrm{HC}$ and $98.28 \% \pm 0.58$ for CFS/ME patients (Fig. 1) as determined by flow cytometry. There was no significant difference in NK cell purity in CFS/ME patients compared with $\mathrm{HC}$.

\section{TRPM3 activity after Pregnenolone sulfate stimulation}

A whole-cell patch-clamp technique was used to measure endogenous TRPM3 activity, enabling a small size of current recordings under voltage-clamp conditions and observation of the typical shape of the TRPM3 currentvoltage relationship ( $I-V$ ) (Fig. 2). We used $100 \mu \mathrm{M}$ PregS to stimulate the channels as TRPM3 is minimally expressed in NK cells (Nguyen et al., 2017; Nguyen et al., 2016). As expected, the ionic current evoked by PregS was relatively small (Fig. 2a and e) in NK cells isolated from $\mathrm{HC}$ and we report a typical $I-V$ of TRPM3, which had a clear outward rectification (Fig. 2b). In contrast, the amplitude of ionic current after PregS stimulation was significantly smaller (Fig. 2c, d) in NK cells from CFS/ME patients than that from $\mathrm{HC}$ (Fig. 2e, $p<0.0001$ ), suggesting impaired TRPM3 channel activity after PregS stimulation in CFS/ME patients.

\section{TRPM3 activity after ononetin modulation}

Ononetin effectively inhibits PregS-evoked $\mathrm{Ca}^{2+}$-influx and ionic currents through TRPM3 channels (Straub et al., 2013). Therefore, to confirm that TRPM3 activity is involved in ionic currents evoked by PregS in NK cells, we next used $10 \mu \mathrm{M}$ ononetin to modulate the channels (Fig. 3). As shown in Fig. 3a, the ionic currents evoked by PregS were effectively inhibited by simultaneous application of ononetin in isolated NK cells from HC. Moreover, the $I-V$ of ononetin sensitive currents was outwardly-rectified and typical for TRPM3 (Fig. 3b). In contrast, ionic currents in the presence of PregS were mostly resistant to ononetin in isolated NK cells from CFS/ME patients (Fig. 3c, d), in comparison with HC (Fig. 3e, f, g $(p=0.0005))$, showing significant loss of the TRPM3 channel activity in CFS/ME patients.

\section{Discussion}

Our previous investigations have proposed that NK cells from CFS/ME patients have significantly reduced expression of TRPM3 and subsequent reduction in intracellular $\mathrm{Ca}^{2+}$ mobilisation compared with HC (Nguyen et al., 2017; Nguyen et al., 2016). This present study used electrophysiological methods to characterise endogenous TRPM3 activity in peripheral NK cells from $\mathrm{HC}$ and CFS/ME patients. We provide evidence suggesting PregS-dependent channel activity for TRPM3 is significantly lower in CFS/ME patients compared with HC. Moreover, ionic currents in CFS/ME patients were resistant to ononetin in the presence of PregS.

The patch clamp technique is regarded as a gold standard for ion channel research and offers direct insight into ion channel properties through the characterization of ion channel activity. In this study, we characterised, for the first time, the TRPM3 ion channel current in isolated human NK cells. We report a significant reduction amplitude of TRPM3 current after PregS stimulation in isolated NK cells from CFS/ME patients compared with $\mathrm{HC}$. This is consistent with our previous findings showing significantly reduced TRPM3 expression as well as significantly reduced intracellular $\mathrm{Ca}^{2+}$ mobilisation in isolated NK cells from CFS/ME patients compared with HC (Nguyen et al., 2017; Nguyen et al., 2016). In addition, we found PregS-evoked ionic currents through TRPM3 channels were significantly modulated by ononetin in isolated $\mathrm{NK}$ cells from $\mathrm{HC}$ compared with CFS/ME patients. Indeed, isolated NK cells from CFS/ME were resistant to ononetin suggesting that PregS may activate non-TRPM3 cationic currents in CFS/ME patients. Alternatively, CFS/ME patients may express different spliced isoforms of TRPM3 that are non-sensitive to ononetin. Although we demonstrate TRPM3 channel activity dysfunction in CFS/ME patients, further investigations are required to elucidate the mechanisms involved in the impaired TRPM3 channel activity as well as the different TRPM3 isoform types that are expressed in NK cells.

Previous electrophysiological investigations have shown that TRPM3 forms an ion channel permeable to $\mathrm{Ca}^{2+}$, sodium $\left(\mathrm{Na}^{+}\right)$, magnesium $\left(\mathrm{Mg}^{2+}\right)$, and manganese $\left(\mathrm{Mn}^{2+}\right)$ (Grimm et al., 2005; Oberwinkler et al., 2005). $\mathrm{Ca}^{2+}$ plays an important role in intracellular signalling pathways, cell differentiation and division, apoptosis, and transcriptional events. In non-excitable cells, such as immune cells, a main $\mathrm{Ca}^{2+}$ entry pathway is known as store-operated $\mathrm{Ca}^{2+}$ entry (SOCE) and some TRP ion channels are associated with this pathway. While the sub-family TRPC have been traditionally associated with this important cellular mechanism (Cheng et al., 2013; Ong et al., 2016; Salido et al., 2009), recent research has also identified TRPM3 as a component for SOCE in 
a

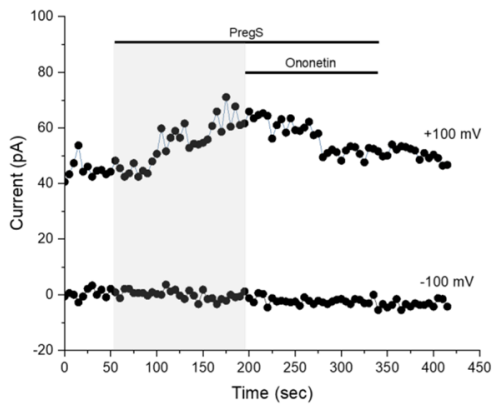

C

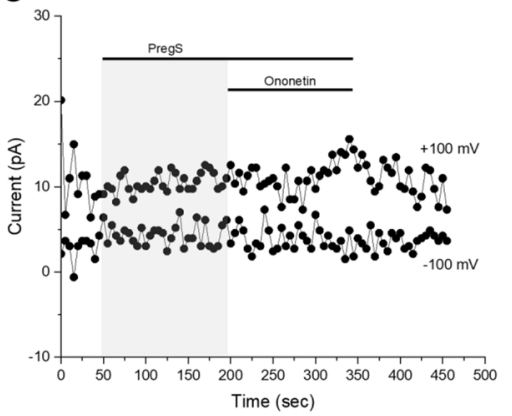

b

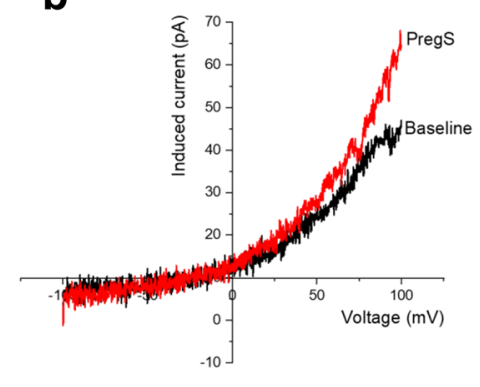

d

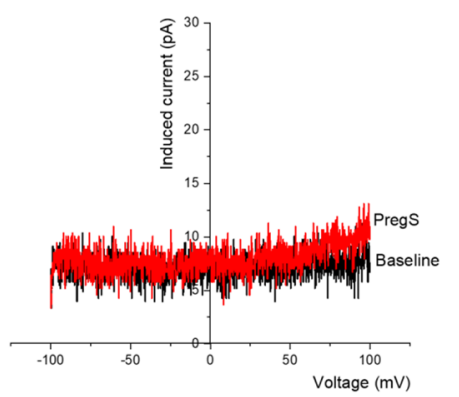

e

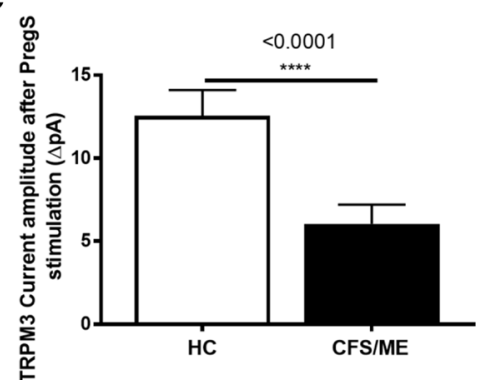

Fig. 2 TRPM3 activity after PregS stimulation. Data were obtained under whole-cell patch clamp conditions. a A representative time-series of current amplitude at $+100 \mathrm{mV}$ and $-100 \mathrm{mV}$ showing the effect of $100 \mu \mathrm{M}$ PregS on ionic currents in isolated NK cells from HC. $\mathbf{b} /-V$ before and after PregS stimulation in a cell corresponding with (a.). c A representative time-series of current amplitude at $+100 \mathrm{mV}$ and $-100 \mathrm{mV}$ showing the effect of $100 \mu \mathrm{M}$ PregS on ionic currents in isolated NK cells from CFS/ME patients. d. I-V before and after PregS stimulation in a cell as shown in (c.). e Bar graphs representing TRPM3 current amplitude at + $100 \mathrm{mV}$ after stimulation with $100 \mu \mathrm{M}$ PregS in CFS/ME patients (N=6; $n=33)$ compared with $H C(N=6 ; n=29)$. Data are represented as mean \pm SEM. Abbreviations: CFS/ME, chronic fatigue syndrome/myalgic encephalomyelitis; HC, healthy control; NK, natural killer

white matter of the central nervous system (CNS) (Papanikolaou et al., 2017). Upon TRPM3 channel activation, changes in $\left[\mathrm{Ca}^{2+}\right]_{\mathrm{i}}$ occur, resulting in the regulation of many biological processes that correspond to an array of cells expressing this channel. TRPM3 is located and linked to vascular smooth muscle contraction, modulation of glucose-induced insulin release from pancreatic islets, detection of noxious heat in dorsal root ganglia and development of epithelial cells of the choroid plexus, as well as function of oligodendrocytes and neurons (Hoffmann et al., 2010; Oberwinkler et al., 2005; Vriens et al., 2011; Wagner et al., 2008). Therefore, dysregulation of TRPM3 family, affecting SOCE and more generally, $\mathrm{Ca}^{2+}$ signalling has significant implications for cell regulatory machinery.
Significant reduction in NK cell cytotoxicity is a consistent feature reported in CFS/ME patients (Brenu et al., 2012; Brenu et al., 2011; Curriu et al., 2013; Hardcastle et al., 2015; Huth et al., 2016a; Klimas et al., 1990; Maher et al., 2005; Natelson et al., 2002; Nijs \& Frémont, 2008; Sharpe et al., 1991; Siegel et al., 2006; Stanietsky \& Mandelboim, 2010). NK cell cytotoxic activity is a $\mathrm{Ca}^{2+}$ dependent process, which drives the intracellular microtubule reorganisation, polarisation of cytoplasmic granules, release of lytic proteins and the creation of the immune synapse (Anasetti et al., 1987; Henkart, 1985). Moreover, $\mathrm{Ca}^{2+}$-dependent cytotoxic processes allow for the production and recruitment of lytic proteins (Voskoboinik et al., 2015). Following cytotoxic granule 
a

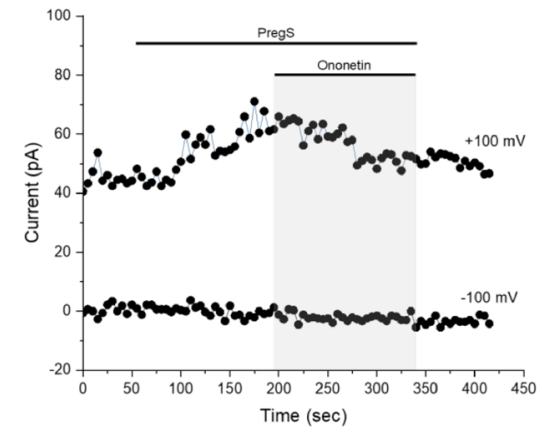

C

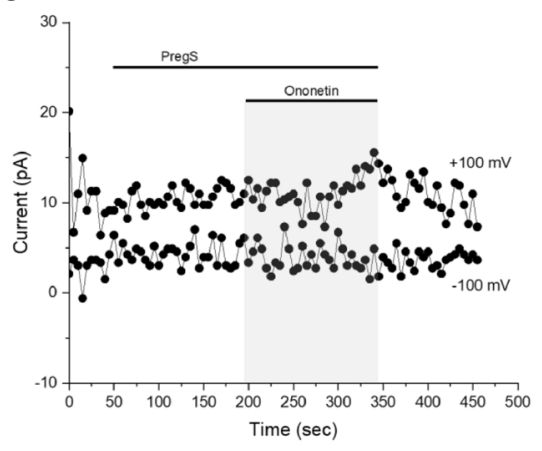

e

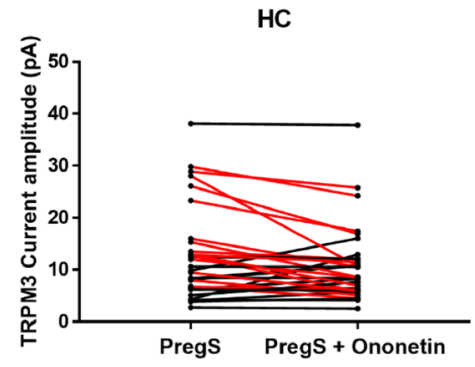

b

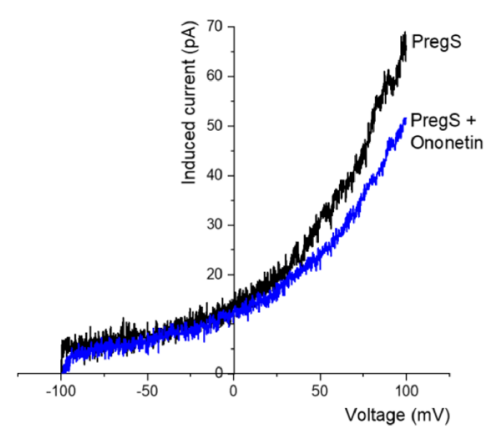

d
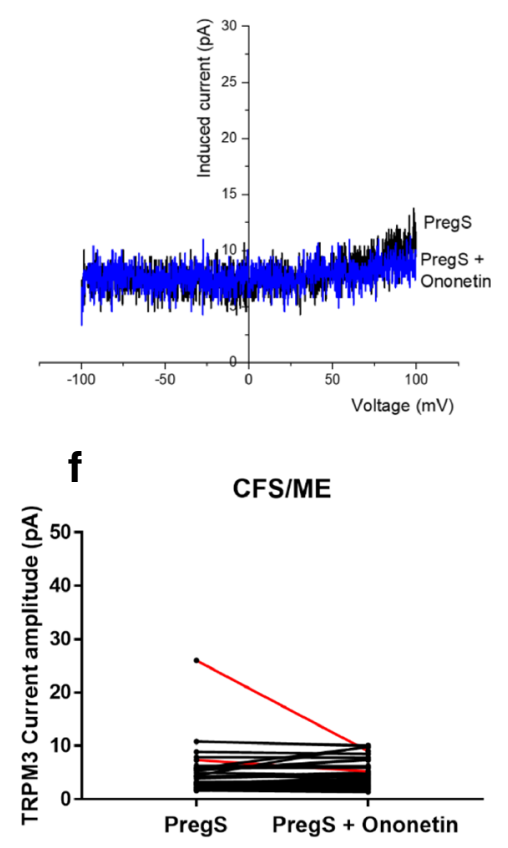

\section{g}

\begin{tabular}{|c|c|c|c|c|}
\hline Data analysed & Sensitive & Insensitive & Total & P Value \\
\hline HC & 16 & 17 & 33 & \\
\hline CFS/ME & 2 & 26 & 28 & \\
\hline Total & 18 & 43 & 61 & 0.0005 \\
\hline
\end{tabular}

\begin{tabular}{|c|c|c|}
\hline Percentage of row total & Sensitive & Insensitive \\
\hline HC & $48.48 \%$ & $\mathbf{5 1 . 5 2 \%}$ \\
\hline CFS/ME & $7.14 \%$ & $\mathbf{9 2 . 8 6 \%}$ \\
\hline
\end{tabular}

Fig. 3 (See legend on next page.) 
(See figure on previous page.)

Fig. 3 TRPM3 activity after ononetin modulation. Data were obtained under whole-cell patch clamp conditions. a. A representative time-series of current amplitude at $+100 \mathrm{mV}$ and $-100 \mathrm{mV}$ showing the effect of $10 \mu \mathrm{M}$ ononetin on ionic currents in the presence of PregS in isolated NK cells from HC. $\mathbf{b} /-V$ before and after application of ononetin in a cell as shown in (a.). $\mathbf{c}$ A representative time-series of current amplitude at $+100 \mathrm{mV}$ and $-100 \mathrm{mV}$ showing the effect of $10 \mu \mathrm{M}$ ononetin on ionic currents in the presence of PregS in isolated NK cells CFS/ME patients. d. $I-V$ before and after application of ononetin in a cell as shown in (c.). e. $\mathbf{f}$ Scatter plots representing change of each current amplitude before and after ononetin application in all NK cells from HC and CFS/ME patients. Each cell represented as red lines had reduction in currents by ononetin. $\mathbf{g}$ Table summarizing data for sensitive and insensitive cells to $10 \mu \mathrm{M}$ ononetin in $\mathrm{HC}(\mathrm{N}=6 ; n=33)$ compared to CFS/ME patients $(N=6 ; n=28)$. Data are analysed with Fisher's exact test. Abbreviations: CFS/ME, chronic fatigue syndrome/myalgic encephalomyelitis; HC, healthy controls

delivery to the immune synapse, the formation of perforin pores and granzyme-induced cell apoptosis are highly dependent on $\mathrm{Ca}^{2+}$ (Orrenius et al., 2003; Voskoboinik et al., 2005). Previous studies have reported impaired $\mathrm{Ca}^{2+}$ signalling in NK cells from CFS/ME patients demonstrated through changes to ERK1/2 and mitogen-activated protein kinase (MAPK) pathways (Chacko et al., 2016; Huth et al., 2016b). CFS/ME patients have significantly decreased ERK1/2 following incubation with $\mathrm{K} 562$ cells (Huth et al., 2016b). ERK1/2 is activated in a Phosphatidylinositol-4,5-bisphosphate 3-kinase $\left(\mathrm{P} 1_{3} \mathrm{~K}\right)$-dependent manner that may also be associated with cytoplasmic $\mathrm{Ca}^{2+}$ ion levels through activation of TRPM3 (Lee et al., 2003). In the absence of phosphatidylinositol 4,5-biphosphate ( $\left.\mathrm{PIP}_{2}\right)$, TRPM3 is not activated, resulting in reduced cytosolic $\mathrm{Ca}^{2+}$ (Tóth et al., 2015). Previous research completed by Nguyen and colleagues reported that the expression of TRPM3 ion channel and $\left[\mathrm{Ca}^{2+}\right]_{\mathrm{i}}$ were significantly reduced in NK cells from CFS/ME patients (Nguyen et al., 2017). In addition, ERK1/2 requires $\mathrm{Ca}^{2+}$ as the final activator to initiate NK cell lysis (Huth et al., 2016b). Changes in $\mathrm{Ca}^{2+}$ signalling may impair cytokine production, including Interferon (IFN) $-\gamma$ and Tumor Necrosis Factor (TNF), therefore interfering with systemic inflammation and anti-tumour responses (Romee et al., 2013). Previous investigations have reported both an increased and decreased inflammatory profile of CFS/ME patients along with reduced IFN- $\gamma$ (Klimas et al., 1990; Lorusso et al., 2009). TRPM3-related $\mathrm{Ca}^{2+}$ dysfunction may then result in a reduction of $\left[\mathrm{Ca}^{2+}\right]_{\mathrm{i}}$, which may lower the function and cytotoxic capacity of the NK cells in CFS/ME patients.

Importantly, TRPM3 ion channels have a role in the detection of heat and in pain transmission in the CNS (Held et al., 2015). TRPM3 has been previously identified as a nociceptor channel involved in acute heat sensing and inflammatory heat hyperalgesia, and thus as a potential target for analgesic treatments (Vriens et al., 2011). Dysregulation of thermoregulatory responses has been reported in CFS/ME patients (Wyller et al., 2007). Generalised pain is a characteristic of CFS/ME and occurs in the absence of overt tissue damage, and this is suggestive of potential CNS impairments (Barnden et al., 2015; Barnden et al., 2016; Shan et al., 2016; Shan et al.,
2017). Our present findings suggest TRPM3 ion channels may be involved in the pathomechansim of CFS/ $\mathrm{ME}$ and hence have a possible role in nociception and thermoregulation.

While this study provides evidence of TRPM3 channel activity dysfunction in CFS/ME patients, this study is not without limitations with the low sample numbers. Indeed, these findings need to be validated in a larger cohort of patients. Additionally, the use of high and single doses of PregS and Ononetin may reduce the drugs specificity and selectivity and consequently modulate other non-TRPM3 cationic currents. Further investigations are also required to assess the impact of PregS and Ononetin on the different TRPM3 isoforms. This could explain the differences observed between $\mathrm{HC}$ and CFS/ME patients as well as within groups. Finally, TRPM4, TRPM5, TRPM2 and TRPM7 surface expression has been reported on B cells, bone marrow cells, splenic cells, lymph node $\mathrm{B}$ cells and $\mathrm{T}$ and mast cells (Zierler et al., 2017), suggesting our recent findings may also be pertinent to other TRPM channel functions and $\mathrm{Ca}^{2+}-$ mediated roles, such as SOCE.

\section{Conclusions}

We have demonstrated impaired TRPM3 activity in CFS/ME patients through electrophysiological investigations in NK cells after modulation with PregS and ononetin. As TRPM3 is widely distributed in the body, particularly brain, eye, cardiovascular system, gastrointestinal system and pancreas, we assert that widespread changes in TRPM3 function in key body systems may contribute to CFS/ME. We suggest changes to $\mathrm{Ca}^{2+}$ ion concentration in the cytosol and intracellular stores result from changes in TRPM3 function, which may impact NK cellular functions. Therefore, $\mathrm{Ca}^{2+}$ signalling pathways could be an alternative therapeutic target in CFS/ME because of their importance in various cellular processes. This investigation confirms the potential role of TRPM3 ion channels in the aetiology and pathomechanism of CFS/ME, and could suggest potential therapeutic targets and/or prognostic markers. 


\section{Abbreviations}

$\left[\mathrm{Ca}^{2+}\right]_{\text {: }}$ Intracellular $\mathrm{Ca}^{2+}$ concentration; ADCC: Antibody-dependent cellular cytotoxicity; BMI: Body Mass Index; Ca ${ }^{2+}$ : Calcium; CCC: Canadian Consensus Criteria; CDC: Centers for Disease Control and Prevention; CFS/ME: Chronic Fatigue Syndrome/Myalgic Encephalomyelitis; CNS: Central nervous system; EDTA: Ethylendiaminetetraacetic acid; ERK: Extracellular signal-regulated kinases; HC: Healthy controls; ICC: International Consensus Criteria; IFN: Interferon; MAPK: Mitogen-activated protein kinase; $\mathrm{Mg}^{2+}$ : Magnesium; $\mathrm{Mn}^{2+}$ : Manganese; $\mathrm{Na}^{+}$: Sodium; NCNED: National Centre for Neuroimmunology and Emerging Diseases; NK: Natural killer; PBMCs: Peripheral blood mononuclear cells; $\mathrm{Pl}_{3} \mathrm{~K}$ : Phosphatidylinositol-4, 5bisphosphate 3-kinase; PIP: Phosphatidylinositol 4,5-bisphosphate; PregS: Pregnenolone sulfate; SF-36: 36-Item Short Form Survey; SNPs: Single nucleotide polymorphisms; SOCE: Store-operated $\mathrm{Ca}^{2+}$ entry; TNF: Tumour necrosis factor; TRP: Transient Receptor Potential; TRPA: Transient Receptor Potential Ankyrin; TRPC: Transient Receptor Potential Canonical; TRPM: Transient Receptor Potential Melastatin; TRPML: Transient Receptor Potential Mucolipin; TRPP: Transient Receptor Potential POlycystin; TRPV: Transient Receptor Potential Vanilloid

\section{Funding}

This study was supported by the Mason Foundation, McCusker Charitable Foundation, Stafford Fox Medical Research Foundation, Mr. Douglas Stutt, Alison Hunter Memorial Foundation, Buxton Foundation, Blake Beckett Trust, Henty Donation, and the Change for ME Charity.

\section{Availability of data and materials}

No data sets were generated for this investigation. All relevant data are present within the manuscript. Data sharing is not applicable to this article under Griffith University Intellectual Property guidelines.

\section{Authors' contributions}

$\mathrm{HC}, \mathrm{KM}, \mathrm{SM}-\mathrm{G}$ and DS designed the study and wrote the manuscript with contributions from other authors. $\mathrm{HC}, \mathrm{NE}$ and $\mathrm{CB}$ performed experiments. $\mathrm{HC}$ and $\mathrm{KM}$ performed data analysis. All authors read and approved the final manuscript.

\section{Ethics approval and consent to participate}

All participants gave written consent to participate.

Ethics approval was under Human Research Ethics Committee Griffith University.

\section{Consent for publication}

All participants gave written consent to publish.

\section{Competing interests}

The authors declare that they have no competing interest.

\section{Publisher's Note}

Springer Nature remains neutral with regard to jurisdictional claims in published maps and institutional affiliations.

\section{Author details University, Chikusa, Nagoya, Japan. \\ Received: 21 May 2018 Accepted: 1 August 2018 Published online: 14 August 2018}

'School of Medical Science, Griffith University, Gold Coast, QLD, Australia. ${ }^{2}$ The National Centre for Neuroimmunology and Emerging Diseases, Menzies Health Institute Queensland, Griffith University, Gold Coast, QLD, Australia. ${ }^{3}$ Laboratory of Cellular Pharmacology, School of Pharmacy, Aichi-Gakuin

\section{References}

Anasetti C, Martin PJ, June CH, Hellstrom KE, Ledbetter JA, Rabinovitch PS, et al. Induction of calcium flux and enhancement of cytolytic activity in natural killer cells by cross-linking of the sheep erythrocyte binding protein (CD2) and the fc-receptor (CD16). J Immunol. 1987;139:1772-9.

Barnden LR, Crouch B, Kwiatek R, Burnet R, Del Fante P. Evidence in chronic fatigue syndrome for severity-dependent upregulation of prefrontal myelination that is independent of anxiety and depression. NMR Biomed. 2015;28:404-13.
Barnden LR, Kwiatek R, Crouch B, Burnet R, Del Fante P. Autonomic correlations with MRI are abnormal in the brainstem vasomotor Centre in chronic fatigue syndrome. Neuroimage Clin. 2016;11:530-7.

Brenu EW, Hardcastle SL, Atkinson GM, van Driel ML, Kreijkamp-Kaspers S, Ashton $\mathrm{K}$, et al. Natural killer cells in patients with severe chronic fatigue syndrome. Auto Immun Highlights. 2013;4:69-80.

Brenu EW, van Driel ML, Staines DR, Ashton KJ, Hardcastle SL, Keane J, et al. Longitudinal investigation of natural killer cells and cytokines in chronic fatigue syndrome/myalgic encephalomyelitis. J Transl Med. 2012;10:88,

Brenu EW, van Driel ML, Staines DR, Ashton KJ, Ramos SB, Keane J, et al. Immunological abnormalities as potential biomarkers in chronic fatigue syndrome/Myalgic encephalomyelitis. J Transl Med. 2011;9:81.

Carruthers BM, van de Sande MI, De Meirleir KL, Klimas NG, Broderick G, Mitchell T, et al. Myalgic encephalomyelitis: international consensus criteria. J Intern Med. 2011:270:327-38.

Chacko A, Staines DR, Johnston SC, Marshall-Gradisnik SM. Dysregulation of protein kinase gene expression in NK cells from chronic fatigue syndrome/ Myalgic encephalomyelitis patients. Gene Regul Syst Bio. 2016;10:85-93.

Cheng KT, Ong HL, Liu X, Ambudkar IS. Contribution and regulation of TRPC channels in store-operated Ca2+ entry. Curr Top Membr. 2013;71:149-79.

Clapham DE, Runnels LW, Strübing C. The TRP ion channel family. Nat Rev Neurosci. 2001;2:387-96.

Cooper MA, Fehniger TA, Caligiuri MA. The biology of human natural killer-cell subsets. Trends Immunol. 2001;22:633-40.

Curriu M, Carrillo J, Massanella M, Rigau J, Alegre J, Puig J, et al. Screening NK-, Band T-cell phenotype and function in patients suffering from Chronic Fatigue Syndrome. J Transl Med. 2013;11:68.

Frühwald J, Camacho Londoño J, Dembla S, Mannebach S, Lis A, Drews A, et al. Alternative splicing of a protein domain indispensable for function of transient receptor potential melastatin 3 (TRPM3) ion channels. J Biol Chem. 2012;287:36663-72.

Fukuda K, Straus SE, Hickie I, Sharpe MC, Dobbins JG, Komaroff A. The chronic fatigue syndrome: a comprehensive approach to its definition and study. International chronic fatigue syndrome study group. Ann Intern Med. 1994;121:953-9.

Gees M, Colsoul B, Nilius B. The Role of Transient Receptor Potential Cation Channels in Ca2+ Signaling. Cold Spring Harb Perspect Biol. 2010 [cited 2018 May 3];2. Available from: https://www.ncbi.nlm.nih.gov/pmc/articles/ PMC2944357/

Grimm C, Kraft R, Schultz G, Harteneck C. Activation of the melastatin-related cation channel TRPM3 by D-erythro-sphingosine [corrected]. Mol Pharmacol. 2005;67:798-805.

Hardcastle SL, Brenu EW, Johnston S, Nguyen T, Huth T, Wong N, et al. Characterisation of cell functions and receptors in Chronic Fatigue Syndrome/ Myalgic Encephalomyelitis (CFS/ME). BMC Immunol. 2015 [cited 2018 May 3];16. Available from: https:/www.ncbi.n/m.nih.gov/pmc/articles/PMC4450981/

Harteneck C. Pregnenolone sulfate: from steroid metabolite to TRP channel ligand. Molecules. 2013;18:12012-28.

Held K, Voets T, Vriens J. TRPM3 in temperature sensing and beyond Temperature (Austin). 2015;2:201-13.

Henkart PA. Mechanism of lymphocyte-mediated cytotoxicity. Annu Rev Immunol. 1985:3:31-58.

Hoffmann A, Grimm C, Kraft R, Goldbaum O, Wrede A, Nolte C, et al. TRPM3 is expressed in sphingosine-responsive myelinating oligodendrocytes. J Neurochem. 2010;114:654-65.

Holakovska B, Grycova L, Jirku M, Sulc M, Bumba L, Teisinger J. Calmodulin and S100A1 protein interact with $\mathrm{N}$ terminus of TRPM3 channel. J Biol Chem. 2012;287:16645-55.

Huth TK, Brenu EW, Ramos S, Nguyen T, Broadley S, Staines D, et al. Pilot study of natural killer cells in chronic fatigue syndrome/Myalgic encephalomyelitis and multiple sclerosis. Scand J Immunol. 2016a;83:44-51.

Huth TK, Staines D, Marshall-Gradisnik S. ERK1/2, MEK1/2 and p38 downstream signalling molecules impaired in CD56dimCD16+ and CD56brightCD16dim/ - natural killer cells in Chronic Fatigue Syndrome/Myalgic Encephalomyelitis patients. J Transl Med. 2016b [cited 2018 May 10];14. Available from: https:// www.ncbi.nlm.nih.gov/pmc/articles/PMC4839077/

Jason LA, Kot B, Sunnquist M, Brown A, Evans M, Jantke R, et al. Chronic fatigue syndrome and Myalgic encephalomyelitis: toward an empirical case definition. Health Psychol Behav Med. 2015;3:82-93.

Johnston S, Brenu EW, Staines D, Marshall-Gradisnik S. The prevalence of chronic fatigue syndrome/ myalgic encephalomyelitis: a meta-analysis. Clin Epidemiol. 2013:5:105-10. 
Kass GE, Orrenius S. Calcium signaling and cytotoxicity. Environ Health Perspect. 1999:107:25-35.

Klimas NG, Salvato FR, Morgan R, Fletcher MA. Immunologic abnormalities in chronic fatigue syndrome. J Clin Microbiol. 1990;28:1403-10.

Lanier LL. Natural killer cell receptor signaling. Curr Opin Immunol. 2003;15:30814.

Lee N, Chen J, Sun L, Wu S, Gray KR, Rich A, et al. Expression and characterization of human transient receptor potential melastatin 3 (hTRPM3). J Biol Chem. 2003;278:20890-7.

Lorusso L, Mikhaylova SV, Capelli E, Ferrari D, Ngonga GK, Ricevuti G. Immunological aspects of chronic fatigue syndrome. Autoimmun Rev. 2009; 8:287-91.

Maher KJ, Klimas NG, Fletcher MA. Chronic fatigue syndrome is associated with diminished intracellular perforin. Clin Exp Immunol. 2005;142:505-11.

Marshall-Gradisnik S, Huth T, Chacko A, Johnston S, Smith P, Staines D. Natural killer cells and single nucleotide polymorphisms of specific ion channels and receptor genes in myalgic encephalomyelitis/chronic fatigue syndrome. Appl Clin Genet. 2016;9:39-47.

Mickle AD, Shepherd AJ, Mohapatra DP. Nociceptive TRP Channels: Sensory Detectors and Transducers in Multiple Pain Pathologies. Pharmaceuticals (Basel). 2016 [cited 2018 May 16];9. Available from: https://www.ncbi.nlm.nih. gov/pmc/articles/PMC5198047/

Moretta L. Dissecting CD56dim human NK cells. Blood. 2010;116:3689-91.

Munoz NM, Leff AR. Highly purified selective isolation of eosinophils from human peripheral blood by negative immunomagnetic selection. Nat Protoc. 2006;1: 2613-20.

Natelson BH, Haghighi MH, Ponzio NM. Evidence for the presence of immune dysfunction in chronic fatigue syndrome. Clin Diagn Lab Immunol. 2002;9:747-52.

Nguyen T, Johnston S, Clarke L, Smith P, Staines D, Marshall-Gradisnik S. Impaired calcium mobilization in natural killer cells from chronic fatigue syndrome/ myalgic encephalomyelitis patients is associated with transient receptor potential melastatin 3 ion channels. Clin Exp Immunol. 2017;187:284-93.

Nguyen T, Staines D, Nilius B, Smith P, Marshall-Gradisnik S. Novel identification and characterisation of transient receptor potential melastatin 3 ion channels on natural killer cells and B lymphocytes: effects on cell signalling in chronic fatigue syndrome/Myalgic encephalomyelitis patients. Biol Res. 2016:49:27.

Nijs J, Frémont M. Intracellular immune dysfunction in myalgic encephalomyelitis/chronic fatigue syndrome: state of the art and therapeutic implications. Expert Opin Ther Targets. 2008;12:281-9.

Nilius B. TRP channels in disease. Biochim Biophys Acta. 2007;1772:805-12.

Nilius B, Voets T. A TRP channel-steroid marriage. Nat Cell Biol. 2008;10:1383-4.

Oberwinkler J, Lis A, Giehl KM, Flockerzi V, Philipp SE. Alternative splicing switches the divalent cation selectivity of TRPM3 channels. J Biol Chem. 2005;280: 22540-8.

Oberwinkler J, Philipp SE. TRPM3. Handb Exp Pharmacol. 2014;222:427-59.

Ong HL, de Souza LB, Ambudkar IS. Role of TRPC channels in store-operated calcium entry. Adv Exp Med Biol. 2016;898:87-109.

Orrenius S, Zhivotovsky B, Nicotera P. Regulation of cell death: the calciumapoptosis link. Nat Rev Mol Cell Biol. 2003;4:552-65.

Papanikolaou M, Lewis A, Butt AM. Store-operated calcium entry is essential for glial calcium signalling in CNS white matter. Brain Struct Funct. 2017;222: 2993-3005

Romee R, Foley B, Lenvik T, Wang Y, Zhang B, Ankarlo D, et al. NK cell CD16 surface expression and function is regulated by a disintegrin and metalloprotease-17 (ADAM17). Blood. 2013;121:3599-608.

Salido GM, Sage SO, Rosado JA. TRPC channels and store-operated ca(2+) entry. Biochim Biophys Acta. 2009:1793:223-30.

Schwarz EC, Qu B, Hoth M. Calcium, cancer and killing: the role of calcium in killing cancer cells by cytotoxic T lymphocytes and natural killer cells. Biochim Biophys Acta. 2013;1833:1603-11.

Shan ZY, Kwiatek R, Burnet R, Del Fante P, Staines DR, Marshall-Gradisnik SM, et al. Progressive brain changes in patients with chronic fatigue syndrome: a longitudinal MRI study. J Magn Reson Imaging. 2016;44:1301-11.

Shan ZY, Kwiatek R, Burnet R, Del Fante P, Staines DR, Marshall-Gradisnik SM, et al. Medial prefrontal cortex deficits correlate with unrefreshing sleep in patients with chronic fatigue syndrome. NMR Biomed. 2017;30

Sharpe MC, Archard LC, Banatvala JE, Borysiewicz LK, Clare AW, David A, et al. A report--chronic fatigue syndrome: guidelines for research. J R Soc Med. 1991; 84:118-21.

Siegel SD, Antoni MH, Fletcher MA, Maher K, Segota MC, Klimas N. Impaired natural immunity, cognitive dysfunction, and physical symptoms in patients with chronic fatigue syndrome: preliminary evidence for a subgroup? J Psychosom Res. 2006;60:559-66.

Stabile H, Nisti P, Morrone S, Pagliara D, Bertaina A, Locatelli F, et al. Multifunctional human CD56 low CD16 low natural killer cells are the prominent subset in bone marrow of both healthy pediatric donors and leukemic patients. Haematologica. 2015;100:489-98.

Stanietsky N, Mandelboim O. Paired NK cell receptors controlling NK cytotoxicity. FEBS Lett. 2010;584:4895-900.

Straub I, Mohr F, Stab J, Konrad M, Philipp S, Oberwinkler J, et al. Citrus fruit and fabacea secondary metabolites potently and selectively block TRPM3. Br J Pharmacol. 2013;168:1835-50.

Thiel G, Müller I, Rössler OG. Signal transduction via TRPM3 channels in pancreatic $\beta$-cells. J Mol Endocrinol. 2013;50:R75-83.

Tóth BI, Oberwinkler J, Voets T. Phosphoinositide regulation of TRPM channels TRPM3 joins the club! Channels (Austin). 2015;10:83-5.

Vivier E, Tomasello E, Baratin M, Walzer T, Ugolini S. Functions of natural killer cells. Nat Immunol. 2008;9:503-10.

Voets T, Talavera K, Owsianik G, Nilius B. Sensing with TRP channels. Nat Chem Biol. 2005;1:85-92.

Voskoboinik I, Thia M-C, Fletcher J, Ciccone A, Browne K, Smyth MJ, et al. Calcium-dependent plasma membrane binding and cell lysis by perforin are mediated through its C2 domain: a critical role for aspartate residues 429 , 435, 483, and 485 but not 491. J Biol Chem. 2005;280:8426-34.

Voskoboinik I, Whisstock JC, Trapani JA. Perforin and granzymes: function, dysfunction and human pathology. Nat Rev Immunol. 2015;15:388-400.

Vriens J, Owsianik G, Hofmann T, Philipp SE, Stab J, Chen X, et al. TRPM3 is a nociceptor channel involved in the detection of noxious heat. Neuron. 2011; 70:482-94.

Wagner TFJ, Loch S, Lambert S, Straub I, Mannebach S, Mathar I, et al. Transient receptor potential M3 channels are ionotropic steroid receptors in pancreatic beta cells. Nat Cell Biol. 2008;10:1421-30.

Wyller VB, Godang K, Mørkrid L, Saul JP, Thaulow E, Walløe L. Abnormal thermoregulatory responses in adolescents with chronic fatigue syndrome: relation to clinical symptoms. Pediatrics. 2007;120:e129-37.

Zierler S, Hampe S, Nadolni W. TRPM channels as potential therapeutic targets against pro-inflammatory diseases. Cell Calcium. 2017;67:105-15.

\section{Ready to submit your research? Choose BMC and benefit from:}

- fast, convenient online submission

- thorough peer review by experienced researchers in your field

- rapid publication on acceptance

- support for research data, including large and complex data types

- gold Open Access which fosters wider collaboration and increased citations

- maximum visibility for your research: over $100 \mathrm{M}$ website views per year

At BMC, research is always in progress.

Learn more biomedcentral.com/submissions 